\title{
A RELATION BETWEEN KILLING TENSOR FIELDS AND NEGATIVE PINCHED RIEMANNIAN MANIFOLDS
}

\author{
GRIGORIOS TSAGAS
}

1. Introduction. Let $M$ be a compact orientable Riemannian manifold. Let $M_{P}$ be the tangent space of the manifold $M$ at the point $P$. We denote by $\langle X, Y\rangle$ and $\|X\|$ the scalar product of two vectors $X, Y \in M_{P}$ and the norm of the vector $X$, respectively, where the scalar product on the tangent space $M_{P}$ is induced by the Riemannian metric of the manifold $M$.

If $X, Y$ are two vectors of the tangent space $M_{P}$, then the curvature tensor field $R$ of the manifold $M$ and the two vectors $X, Y$ induce an endomorphism $R(X, Y)$ of $M_{P}$ into $M_{P}$. If $X, Y, Z, T$ are four vectors of $M_{P}$, then the Riemannian curvature tensor $R_{1}$ at $P$ can be considered as a quadrilinear mapping $R_{1}: M_{P} \times M_{P} \times M_{P} \times M_{P} \rightarrow R$ which is defined by $R_{1}:(X, Y, Z, T) \rightarrow\langle R(X, Y) Z, T\rangle$. Let $\lambda$ be a plane of the tangent space $M_{P}$ which is spanned by two linearly independent vectors $X, Y \in M_{P}$. The sectional curvature of the plane $\lambda$ is given by

$$
\sigma(\lambda)=\sigma(X, Y)=-\frac{\langle R(X, Y) X, Y\rangle}{\|X\|^{2}\|Y\|^{2}-\langle X, Y\rangle^{2}} .
$$

We assume that the Riemannian manifold is compact orientable and negative $\delta$-pinched, that means its sectional curvature $\sigma(\lambda)$ satisfies the inequalities

$$
-\Lambda \leqq \sigma(\lambda) \leqq-\Lambda \delta,
$$

for every $\lambda \in M_{P}$ and $\forall P \in M$.

We can normalize the metric on the manifold $M$ such that the above inequalities become

$$
-1 \leqq \sigma(\lambda) \leqq-\delta .
$$

A Riemannian manifold $M$, whose sectional curvature satisfies the above inequalities, is called negative $\delta$-pinched.

Now, our results can be stated as follows: Let $M$ be a compact orientable negative $\delta$-pinched manifold. If the dimension of $M$ is even, $n=2 m($ resp. odd,$n=2 m+1)$ and $\delta>1 / 4($ resp. $\delta>2(m-1) /(8 m-5)$ ), then there exists neither Killing tensor field of order 2 nor conformal Killing tensor field of order 2 .

2. Let $M$ be a compact orientable negative $\delta$-pinched manifold.

Received by the editors October 30, 1968. 
We obtain a point $P$ of the manifold $M$ and consider a normal coordinate system on $M$ with origin the point $P$.

We also consider an orthonormal basis $\left\{X_{1}, \cdots, X_{n}\right\}$ of the tangent space $M_{P}$. If $\left\{X_{i}, X_{j}, X_{k}, X_{l}\right\}$ is a set of four vectors of the orthonormal basis $\left\{X_{1}, \cdots, X_{n}\right\}$, then the following formulas hold:

$$
\left\langle R\left(X_{i}, X_{j}\right) X_{k}, X_{l}\right\rangle=R_{i j k l}, \quad \sigma\left(X_{i}, X_{j}\right)=\sigma_{i j}=-R_{i j i j},
$$

where $R_{i j k l}$ are the components of the Riemannian curvature.

If we apply the same technique as in [1, pp. 67-69], we obtain the following inequalities:

$$
\left|R_{i j i k}\right| \leqq(1-\delta) / 2, \quad\left|R_{i j k l}\right| \leqq 2(1-\delta) / 3, \quad i \neq j \neq k \neq l,
$$

because all the computations made in [1] for the sectional curvature ranging $\left(\frac{1}{4}, 1\right]$ if $n=2 m$, or $(2(m-1) /(9 m-5), 1]$ if $n=2 m+1$ are valid without any change of the curvature ranging in any interval $[a, b]$.

3. Let $\xi=\left\{\xi\left(X_{i}, X_{j}\right)=\xi_{i j}\right\}$ be an exterior 2-form. This exterior 2 -form is called a Killing 2 -form if it satisfies the relation:

$$
\nabla_{X} \xi(Y, Z)=-\nabla_{Y} \xi(X, Z), \quad \forall X, Y, Z \in T(M) .
$$

We consider the quadratic form $F(\xi)$ given by $[2$, p. 62]:

$$
F(\xi)=\sum_{i j i_{2}} R_{i j} \xi^{i i_{2}} \xi_{i_{2}}^{j}+\frac{1}{2} \sum_{i j k l} R_{i j k l} \xi^{i j} \xi^{k l} .
$$

If the manifold is even dimension $n=2 m$ (resp. odd dimension $n=2 m+1)$ and $\delta>1 / 4$ (resp. $\delta>2(m-1) /(8 m-5)$ ), then by means of the second of (2.1) and (2.2) with the same method as in $[1, \mathrm{p} .70]$, we have $F(\xi)<0$. It is well known $[2$, p. 67], if $F(\xi)<0$, it implies $\xi=0$.

Therefore, we can state the following theorem.

THEOREM I. Let $M$ be a compact orientable negative $\delta$-pinched manifold. If the dimension of the manifold is even $n=2 m$ (resp. odd $n$ $=2 m+1)$ and $\delta>1 / 4($ resp. $\delta>2(m-1) /(8 m-5))$, then there exists no Killing tensor field of order 2 on the manifold $M$.

4. Let $n=\left\{n\left(X_{i}, X_{j}\right)=n_{i}, n_{2}\right\}$ be an exterior 2 -form on the manifold $M$. This exterior 2 -form is called a conformal Killing 2 -form, if it satisfies the following conditions $[2$, p. 73]:

$$
\nabla_{j} n_{i i_{2}}+\nabla_{i} n_{j i_{2}}=2 \beta_{i_{2}} g_{i j}, \quad \beta_{i_{2}}=g^{i j} \nabla_{j} n_{i i_{2}} / n .
$$

We can prove with the same technique as in $\S 3$. If the manifold $M$ 
is compact orientable and negative $\delta$-pinched, the dimension of the manifold is even $n=2 m$, (resp. odd $n=2 m+1$ ), and $\delta>1 / 4$ (resp. $\delta$ $>2(m-1) /(8 m-5))$, then the quadratic form $F(n)$ is negative definite.

From the above and the known theorem [2, p. 73], we obtain the following theorem:

TheOREM II. Let $M$ be a compact orientable negative $\delta$-pinched Riemannian manifold. If the dimension of the manifold is even $n=2 m$ (resp. odd $n=2 m+1)$ and $\delta>1 / 4$ (resp. $\delta>2(m-1) /(8 m-5))$, then there exists no conformal Killing tensor field of order 2 on the manifold.

I wish to express here my thanks to Professor S. Kobayashi for many good ideas I obtained from conversation with him.

\section{REFERENCES}

1. M. Berger, Sur quelques variétés Riemanniennes suffisamment pincées, Bull. Soc. Math. France 88 (1960), 57-71.

2. K. Yano and S. Bochner, Curvature and Betti numbers, Ann. of Math. Studies, No. 32, Princeton Univ. Press, Princeton, N. J., 1953.

University of California, Berkeley 